\title{
A Generalized Symbolic Thomas Algorithm for Solving Doubly Bordered $k$-Tridiagonal Linear Systems
}

\author{
Nermeen Shehab $^{1}{ }^{*}$, Moawwad El-Mikkawy ${ }^{2}$, Mohammed El-Shehawy ${ }^{1}$ \\ ${ }^{1}$ Mathematics Department, Faculty of Science, Damietta University, Damietta, Egypt \\ ${ }^{2}$ Mathematics Department, Faculty of Science, Mansoura University, Mansoura, Egypt \\ Email: ${ }^{*}$ nermeen shehab87@yahoo.com, m elmikkawy@yahoo.com, melshehawey@yahoo.com
}

Received 16 June 2015; accepted 26 September 2015; published 29 September 2015

Copyright (C) 2015 by authors and Scientific Research Publishing Inc.

This work is licensed under the Creative Commons Attribution International License (CC BY).

http://creativecommons.org/licenses/by/4.0/

(c) (;) Open Access

\section{Abstract}

In the current paper, the authors present a symbolic algorithm for solving doubly bordered $k$-tridiagonal linear system having $n$ equations and $n$ unknowns. The proposed algorithm is derived by using partition together with UL factorization. The cost of the algorithm is $O(n)$. The algorithm is implemented using the computer algebra system, MAPLE. Some illustrative examples are given.

\section{Keywords}

Doubly Bordered $\boldsymbol{k}$-Tridiagonal Matrix, UL Factorization, DETGDBTRI Algorithm, Thomas Algorithm, Computer Algebra Systems (CAS)

\section{Introduction}

The general tridiagonal matrixtakes the form:

$$
T_{n}=\left[\begin{array}{cccccc}
d_{1} & a_{1} & 0 & \ldots & \ldots & 0 \\
b_{2} & d_{2} & a_{2} & \ddots & & \vdots \\
0 & b_{3} & d_{3} & a_{3} & \ddots & \vdots \\
\vdots & \ddots & \ddots & \ddots & \ddots & 0 \\
\vdots & & \ddots & \ddots & \ddots & a_{n-1} \\
0 & \ldots & \ldots & 0 & b_{n} & d_{n}
\end{array}\right]
$$

\footnotetext{
*Corresponding author.
}

How to cite this paper: Shehab, N., El-Mikkawy, M. and El-Shehawy, M. (2015) A Generalized Symbolic Thomas Algorithm for Solving Doubly Bordered k-Tridiagonal Linear Systems. Journal of Applied Mathematics and Physics, 3, 1199-1206. 
Such matrices arise in many applications, such as boundary value problems, parallel computing, telecommunication system analysis, interpolation with splines and solution of differential equations using finite differences. Research area on these types of matrices is very active and has recently attracted the attention of many researchers. The interested reader may refer to [1]-[4].

Recently, researchers have begun considering the $k$-tridiagonal matrix as a generalization of the special matrixin (1).

The general $k$-tridiagonal matrix takes the form:

$$
T_{n}^{(k)}=\left[\begin{array}{ccccccccc}
d_{1} & 0 & \ldots & 0 & a_{1} & 0 & \ldots & \ldots & 0 \\
0 & d_{2} & 0 & \cdots & 0 & a_{2} & \ddots & & \vdots \\
\vdots & 0 & d_{3} & \ddots & & \ddots & a_{3} & \ddots & \vdots \\
0 & \vdots & \ddots & \ddots & \ddots & & \ddots & \ddots & 0 \\
b_{k+1} & 0 & & \ddots & \ddots & \ddots & & \ddots & a_{n-k} \\
0 & b_{k+2} & \ddots & & \ddots & d_{n-k} & \ddots & & 0 \\
\vdots & \ddots & \ddots & \ddots & & \ddots & \ddots & \ddots & \vdots \\
\vdots & & \ddots & b_{n-1} & \ddots & & \ddots & d_{n-1} & 0 \\
0 & \ldots & \ldots & 0 & b_{n} & 0 & \ldots & 0 & d_{n}
\end{array}\right]
$$

For example,

$$
T_{7}^{(4)}=\left[\begin{array}{ccccccc}
d_{1} & 0 & 0 & 0 & a_{1} & 0 & 0 \\
0 & d_{2} & 0 & 0 & 0 & a_{2} & 0 \\
0 & 0 & d_{3} & 0 & 0 & 0 & a_{3} \\
0 & 0 & 0 & d_{4} & 0 & 0 & 0 \\
b_{5} & 0 & 0 & 0 & d_{5} & 0 & 0 \\
0 & b_{6} & 0 & 0 & 0 & d_{6} & 0 \\
0 & 0 & d_{7} & 0 & 0 & 0 & d_{7}
\end{array}\right]
$$

The importance of such matrices could be shown clearly in the last few years. For more details see, for instance, [5]-[8].

In this paper, we are going to focus on the doubly bordered $k$-tridiagonal matrix, here after will be referred to as $k$-DBT, which has the form:

$$
A_{n}^{(k)}=\left[\begin{array}{ccccccccc}
d_{1} & h_{1} & h_{2} & \cdots & \cdots & h_{k+1} & \cdots & \cdots & h_{n-1} \\
v_{1} & d_{2} & 0 & \cdots & 0 & a_{2} & 0 & \cdots & 0 \\
v_{2} & 0 & d_{3} & 0 & & \ddots & a_{3} & \ddots & \vdots \\
\vdots & \vdots & 0 & \ddots & \ddots & & \ddots & \ddots & 0 \\
\vdots & 0 & & \ddots & \ddots & \ddots & & \ddots & a_{n-k} \\
v_{k+1} & b_{k+2} & \ddots & & \ddots & d_{n-k} & \ddots & & 0 \\
\vdots & 0 & \ddots & \ddots & & \ddots & \ddots & \ddots & \vdots \\
\vdots & \vdots & \ddots & b_{n-1} & \ddots & & \ddots & d_{n-1} & 0 \\
v_{n-1} & 0 & \cdots & 0 & b_{n} & 0 & \cdots & 0 & d_{n}
\end{array}\right]
$$

We can hold the view that the above matrix is a natural extension of the k-tridiagonal matrix in (2). In a partitioned form, the matrix $A_{n}^{(k)}$ in (4) can be written as: 


$$
\left[\begin{array}{c:cccccccc}
d_{1} & h_{1} & h_{2} & \ldots & \cdots & h_{k+1} & \ldots & \ldots & h_{n-1} \\
\hdashline v_{1} & d_{2} & 0 & \cdots & 0 & a_{2} & 0 & \cdots & 0 \\
v_{2} & 0 & d_{3} & 0 & & \ddots & a_{3} & \ddots & \vdots \\
\vdots & \vdots & 0 & \ddots & \ddots & & \ddots & \ddots & 0 \\
\vdots & 0 & & \ddots & \ddots & \ddots & & \ddots & a_{n-k} \\
v_{k+1} & b_{k+2} & \ddots & & \ddots & d_{n-k} & \ddots & & 0 \\
\vdots & 0 & \ddots & \ddots & & \ddots & \ddots & \ddots & \vdots \\
\vdots & \vdots & \ddots & b_{n-1} & \ddots & & \ddots & d_{n-1} & 0 \\
v_{n-1} & 0 & \cdots & 0 & b_{n} & 0 & \cdots & 0 & d_{n}
\end{array}\right]=\left[\begin{array}{c|c}
d_{1} & \boldsymbol{h}^{t} \\
\hdashline \boldsymbol{v} & T_{n-1}^{(k)}
\end{array}\right]
$$

where $\boldsymbol{h}^{t}=\left[\begin{array}{lllll}h_{1} & h_{2} & \ldots & h_{n-2} & h_{n-1}\end{array}\right], \boldsymbol{v}=\left[\begin{array}{lllll}v_{1} & v_{2} & \ldots & v_{n-2} & v_{n-1}\end{array}\right]^{t}, \boldsymbol{h}, \boldsymbol{v} \in \mathbb{R}^{(n-1) \times 1}$ and

$$
T_{n-1}^{(k)}=\left[\begin{array}{cccccccc}
d_{2} & 0 & \cdots & 0 & a_{2} & 0 & \cdots & 0 \\
0 & d_{3} & 0 & & \ddots & a_{3} & \ddots & \vdots \\
\vdots & 0 & \ddots & \ddots & & \ddots & \ddots & 0 \\
0 & & \ddots & \ddots & \ddots & & \ddots & a_{n-k} \\
b_{k+2} & \ddots & & \ddots & d_{n-k} & \ddots & & 0 \\
0 & \ddots & \ddots & & \ddots & \ddots & \ddots & \vdots \\
\vdots & \ddots & b_{n-1} & \ddots & & \ddots & d_{n-1} & 0 \\
0 & \cdots & 0 & b_{n} & 0 & \cdots & 0 & d_{n}
\end{array}\right] .
$$

Throughout this paper, the word "simplify" means to simplify the algebraic expression under consideration to its simplest rational form. Also, $\lambda$ is a formal parameter which can be treated as a symbolic name whose actual value is 0 as we will see later.

The present paper is organized as follows. In the next section, numeric and symbolic algorithms for evaluating the $k$-DBT determinant are constructed. The UL factorization of doubly bordered $k$-tridiagonal matrix is also considered. Finally, the solution of the linear system whose coefficient matrix is of type $k$-DBT is proposed. In Section 4, some illustrative examples are given.

\section{Generalization of the DETGDBTRI Algorithm}

In order to factor the $k$-tridiagonal matrix in (6), it is advantageous to introduce the $(n-1)$ quantities associated with the matrix $T_{n-1}^{(k)}$ as follows:

$$
e_{i}= \begin{cases}d_{i} & \text { if } i=n, n-1, \cdots, n-k+1 \\ d_{i}-a_{i} \frac{b_{i+k}}{e_{i+k}} & \text { if } i=n-k, n-k-1, \cdots, 2\end{cases}
$$

Now, consider the following theorem whose proof will be omitted.

Theorem (1):

The Doolittle $U_{T}^{(k)} L_{T}^{(k)}$ of the matrix $T_{n-1}^{(k)}$ takes the form:

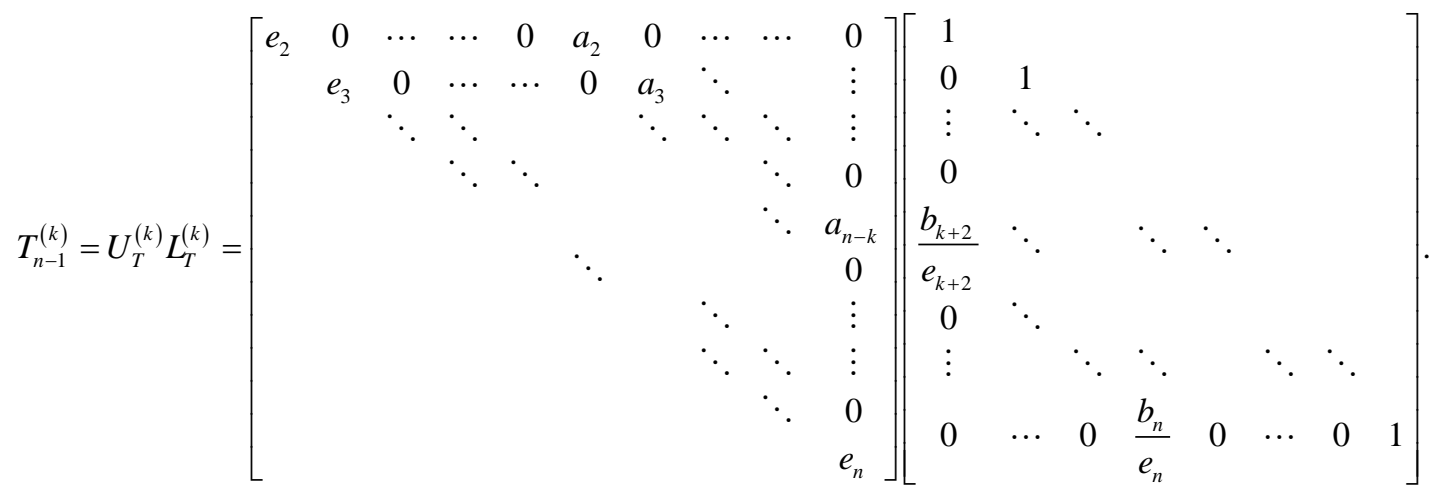


At this point, it should be mentioned that the above factorization in (8) is always possible even if the matrix $T_{n-1}^{(k)}$ is singular.

Armed with the partitioned form of $A^{(k)}$ introduced in (4), we can construct the $U_{A}^{(k)} L_{A}^{(k)}$ factorization of this matrix as follows:

$$
A^{(k)}=\left[\begin{array}{c:c}
d_{1} & \boldsymbol{h}^{t} \\
\hdashline \boldsymbol{v} & T_{n-1}^{(k)}
\end{array}\right]=\left[\begin{array}{c:c}
e_{1} & \boldsymbol{q}^{t} \\
\hdashline \mathbf{0} & U_{T}^{(k)}
\end{array}\right]\left[\begin{array}{c:c}
1 & \mathbf{0} \\
\hdashline \boldsymbol{p} & L_{T}^{(k)}
\end{array}\right]
$$

where $\boldsymbol{q}^{t}=\left[\begin{array}{lllll}q_{1} & q_{2} & \cdots & q_{n-2} & q_{n-1}\end{array}\right], \boldsymbol{p}=\left[\begin{array}{lllll}p_{1} & p_{2} & \cdots & p_{n-2} & p_{n-1}\end{array}\right]^{t}, L_{T}^{(k)}$ and $U_{T}^{(k)}$ are given in (8).

By using the above equation, we see that the following four systems of equations are necessarily satisfied

$$
\begin{gathered}
e_{1}+\boldsymbol{q}^{t} \boldsymbol{p}=d_{1}, \\
\boldsymbol{h}^{t}=\boldsymbol{q}^{t} L_{T}^{(k)}, \\
\boldsymbol{v}=U_{T}^{(k)} \boldsymbol{p},
\end{gathered}
$$

and

$$
T_{n-1}^{(k)}=U_{T}^{(k)} L_{T}^{(k)}
$$

Solving (10)-(12) for $e_{1}, \boldsymbol{q}$ and $\boldsymbol{p}$ yields:

$$
\begin{gathered}
q_{i}=\left\{\begin{array}{cc}
h_{i} & \text { if } i=n-1, n-2, \cdots, n-k, \\
h_{i}-z_{i+k+1} q_{i+k} & \text { if } i=n-k-1, n-k-2, \cdots, 1 .
\end{array}\right. \\
p_{i}= \begin{cases}\frac{v_{i}}{e_{i+1}} & \text { if } i=n-1, n-2, \cdots, n-k, \\
\left(v_{i}-a_{i+1} p_{i+k}\right) / e_{i+1} & \text { if } i=n-k-1, n-k-2, \cdots, 1 .\end{cases}
\end{gathered}
$$

and

$$
e_{1}=d_{1}-\sum_{i=1}^{n-1} q_{i} p_{i}
$$

where $z_{i}=\frac{b_{i}}{e_{i}}, i=k+2, \cdots, n$.

We may now formulate the following algorithm for evaluating $\operatorname{det}\left(A^{(k)}\right)$ of the matrix $A^{(k)}$ in (4).

Algorithm 2.1. A numeric algorithm for computing $\operatorname{det}\left(A^{(k)}\right)$ in (4).

To compute the determinant of the $k$-DBT matrix $A^{(k)}$ in (4), we may proceed as follows:

INPUT: Order of the matrix, $n$, the value of $k$ and vectors $\boldsymbol{a}, \boldsymbol{d}, \boldsymbol{b}, \boldsymbol{h}, \boldsymbol{v}$.

OUTPUT: the determinant of the matrix $A^{(k)}$ in (4)

Step 1: For $i=n, n-1, \cdots, n-k+1$

set: $e_{i}=d_{i}$

End do

Step 2: For $i=k+2, k+3, \cdots, n$ do 


$$
\text { set: } z_{i}=\frac{b_{i}}{e_{i}}
$$

End do.

Step 3: For $i=n-k, n-k-1, \cdots, 2$ do compute and simplify:

$$
e_{i}=d_{i}-a_{i} z_{i+k}
$$

End do.

Step 4: Compute and simplify $e_{1}$ using (16)

Step 5: $\operatorname{Det}\left(A^{(k)}\right)=\prod_{r=1}^{n} e_{r}$.

As can be easily seen, Algorithm (2.1) breaks down if any $e_{i}=0$ for some $i \in\{1,2, \ldots, n\}$ The following symbolic algorithm is developed in order to remove the case where the numeric algorithm fails.

Algorithm 2.2 A symbolic algorithm for computing $\operatorname{det}\left(A^{(k)}\right)$ in (4).

To compute the determinant of the $k$-DBT matrix $A^{(k)}$ in (4), we may proceed as follows:

INPUT: Order of the matrix, $n$, the value of $k$ and vectors $\boldsymbol{a}, \boldsymbol{d}, \boldsymbol{b}, \boldsymbol{h}, \boldsymbol{v}$.

OUTPUT: the determinant of the matrix $A^{(k)}$ in (4)

Step 1: For $i=n, n-1, \cdots, n-k+1$

set: $e_{i}=d_{i}$ If any $e_{i}=0$ then $e_{i}=\lambda$ end if

( $\lambda$ is just a symbolic name)

End do

Step 2: For $i=k+2, k+3, \cdots, n$ do

$$
\text { set: } z_{i}=\frac{b_{i}}{e_{i}}
$$

End do.

Step 3: For $i=n-k, n-k-1, \cdots, 2$ do

compute and simplify:

$e_{i}=d_{i}-a_{i} z_{i+k}$. If $e_{i}=0$ then $e_{i}=\lambda$.

End do.

Step 4: Compute and simplify $e_{1}$ using (16).

Step 5: Compute and simplify:

$$
p(\lambda)=\prod_{r=1}^{n} e_{r} .
$$

Step 6: $\operatorname{Det}\left(A^{(k)}\right)=p(0)$.

The Algorithm 2.2 will be referred to as $\boldsymbol{k}$-DETGDBTRI algorithm. It is a natural extension of the DETGDBTRI algorithm presented in [11]. 


\section{Solving Linear System of Equations with Coefficient Matrix of Typek-DBT}

In this section, we introduce a symbolic algorithm for solving $k$-DBT linear systems of the form:

$$
A^{(k)} \boldsymbol{x}=\boldsymbol{f}
$$

where $\quad \boldsymbol{x}=\left[\begin{array}{llll}x_{1} & x_{2} & \cdots & x_{n}\end{array}\right]^{t}, \boldsymbol{f}=\left[\begin{array}{llll}f_{1} & f_{2} & \cdots & f_{n}\end{array}\right]^{t}$ and $A^{(k)}$ introduced in (4).

Algorithm 3.1 (Asymbolic algorithm for solving linear systems of $\boldsymbol{k}$-DBT matrix
To solve a general $k$-DBT linear system of the form (17), we may proceed as follows:
INPUT: The components, $a_{i}, b_{i}, d_{i}, h_{i}, v_{i}$ and $f_{i}$
OUTPUT: The solution vector $\boldsymbol{x}=\left[x_{1} x_{2} \cdots x_{n}\right]^{t}$ of the linear system in (17).
Step 1: Use the $\boldsymbol{k}$-DETGDBTRI algorithm to compute and simplify $e_{i}$ for $i=1,2, \cdots$
Step 2: Compute and simplify $q_{i}, i=1, \cdots, n-1$ using (14).
Step 3: Compute and simplify $p_{i}, i=1, \cdots, n-1$ using (15).
Step 4: Compute $w_{1}, w_{2}, \cdots, w_{n}$ using:
\[ w_{i}= \begin{cases}\left(f_{i}-a_{i} w_{i+k}\right) / e_{i} & \text { if } i=n, \cdots, n-k+1 \\ \left(f_{1}-\sum_{r=1}^{n-1} q_{r} w_{r+1}\right) / e_{1} & \text { if } i=1\end{cases} \]

Step 5: The solution vector $\mathrm{x}$ of the system (17) is given by

$$
x_{i}= \begin{cases}w_{1} & \text { if } i=1 \\ w_{i}-p_{i-1} x_{1} & \text { if } i=2, \cdots, k+1 \\ w_{i}-p_{i-1} x_{1}-z_{i} x_{i-k} & \text { if } i=k+2, \cdots, n\end{cases}
$$

evaluated at $\lambda=0$.

The Algorithm (3.2) will be referred to as $k$-DBTLSys algorithm. The total computational cost of the $k$ DBTLSys algorithm is $19 n-11 k-18$ in terms of total number of flops, where each flop represents one of the four basic arithmetic floating point operations.

A maple code based on algorithm 3.1 is available upon request from the authors.

The following four remarks are given in order:

Remark 1. If $k=1, p_{i}=q_{i}=0, i=2,3, \cdots, n-1$, then we have the DETGTRI algorithm in [9].

Remark 2. If $k=1, p_{i}=q_{i}=0, i=2,3, \cdots, n-2$, then we have the PERTRI algorithm in [10].

Remark 3. If $1<k<n, p_{i}=q_{i}=0, i=2,3, \cdots, n-1$, then we have the $k$-DETGTRI algorithm in [8].

Remark 4. If $k=1, p_{i} \neq 0, q_{i} \neq 0$ for some $i=2,3, \cdots, n-1$, then we have the DBTLSys algorithm in [11].

\section{Illustrative Examples}

Notice that in the following examples, blank elements in the matrices are zeros.

Example 4.1. Consider the following k-DBT linear system:

$$
\left[\begin{array}{cccccc}
1 & 2 & 1 & 4 & 5 & 3 \\
3 & 2 & 0 & 0 & 3 & 0 \\
2 & 0 & 1 & 0 & 0 & 2 \\
-3 & 0 & 0 & 2 & 0 & 0 \\
5 & 2 & 0 & 0 & 1 & 0 \\
1 & 0 & 1 & 0 & 0 & 2
\end{array}\right]\left[\begin{array}{l}
x_{1} \\
x_{2} \\
x_{3} \\
x_{4} \\
x_{5} \\
x_{6}
\end{array}\right]=\left[\begin{array}{c}
15 \\
7 \\
29 \\
-13 \\
25 \\
24
\end{array}\right]
$$


Solution: In this example, we have $n=6$ and $k=3$.

Applying the $k$-DBTLSys algorithm gives:

- $e_{1}=\frac{9 \lambda+1}{2 \lambda}, e_{2}=-4, e_{3}=\lambda, e_{4}=2, e_{5}=1, e_{6}=2$. Hence $\operatorname{Det}(A)=-8$. Thus, A is nonsingular (step 1 ).

- $\boldsymbol{q}^{t}=\left[\begin{array}{lllll}-8 & -1 / 2 & 4 & 5 & 3\end{array}\right]$ (step 2).

- $\boldsymbol{p}=\left[\begin{array}{lllll}3 & 1 / \lambda & -3 / 2 & 5 & 1 / 2\end{array}\right]^{t}$ (step 3).

- $w_{1}=\frac{32 \lambda+5}{9 \lambda+1}, w_{2}=17, w_{3}=\frac{5}{\lambda}, w_{4}=\frac{-13}{2}, w_{5}=25, w_{6}=12$ (step 4).

- $x_{1}=\left.\frac{32 \lambda+5}{9 \lambda+1}\right|_{\lambda=0}=5, x_{2}=\left.\frac{57 \lambda+2}{9 \lambda+1}\right|_{\lambda=0}=2, x_{3}=\left.\frac{13}{9 \lambda+1}\right|_{\lambda=0}=13, x_{4}=\left.\frac{-(21 \lambda-2)}{2(9 \lambda+1)}\right|_{\lambda=0}=1$,

$x_{5}=\left.\frac{-(49 \lambda+4)}{9 \lambda+1}\right|_{\lambda=0}=-4, x_{6}=\left.\frac{92 \lambda+3}{9 \lambda+1}\right|_{\lambda=0}=3$ (step 5).

The solution is $\boldsymbol{x}=\left[\begin{array}{llllll}5 & 2 & 13 & 1 & -4 & 3\end{array}\right]^{t}$.

Example 4.2. Consider the following k-DBT linear system:

$$
\left[\begin{array}{cccccccccc}
4 & 1 & -3 & -1 & 0 & 2 & 1 & 1 & -1 & 2 \\
1 & 1 & & & & & 2 & & & \\
-1 & & 3 & & & & & -2 & & \\
2 & & & -1 & & & & & 2 & \\
0 & & & & 2 & & & & & -2 \\
2 & & & & & 1 & & & & \\
3 & 1 & & & & & 5 & & & \\
4 & & -1 & & & & & -1 & & \\
2 & & & 1 & & & & & 2 & \\
5 & & & & -1 & & & & & -2
\end{array}\right]\left[\begin{array}{c}
x_{1} \\
x_{2} \\
x_{3} \\
x_{4} \\
x_{5} \\
x_{6} \\
x_{7} \\
x_{8} \\
x_{9} \\
x_{10}
\end{array}\right]=\left[\begin{array}{l}
4 \\
4 \\
0 \\
3 \\
0 \\
3 \\
9 \\
2 \\
5 \\
2
\end{array}\right]
$$

Solution: In this example, we have $n=10$ and $k=5$.

Applying the $k$-DBTLSys algorithm yields:

- $\boldsymbol{e}=\left[\begin{array}{llllllllll}4 / 5 & 3 / 5 & 5 & -2 & 3 & 1 & 5 & -1 & 2 & -2\end{array}\right]$ (step 1).

- $\boldsymbol{q}^{t}=\left[\begin{array}{lllllllll}4 / 5 & -4 & -1 / 2 & -1 & 2 & 1 & 1 & -1 & 2\end{array}\right]$ (step 2).

- $\boldsymbol{p}=\left[\begin{array}{lllllllll}-1 / 3 & -9 / 5 & 0 & -5 / 3 & 2 & 3 / 5 & -4 & 1 & -5 / 2\end{array}\right]^{t} \quad$ (step 3).

- $\boldsymbol{w}=\left[\begin{array}{llllllllll}-3 / 2 & 2 / 3 & -4 / 5 & 1 & -2 / 3 & 3 & 9 / 5 & -2 & 5 / 2 & -1\end{array}\right]^{t}$ (step 4).

The solution is $\boldsymbol{x}=\left[\begin{array}{llllllllll}-3 / 2 & 1 / 6 & -7 / 2 & 1 & -19 / 6 & 6 & 8 / 3 & -9 / 2 & 7 / 2 & -19 / 6\end{array}\right]^{t} \quad$ (step 5).

Example 4.3. Consider the following k-DBT linear system:

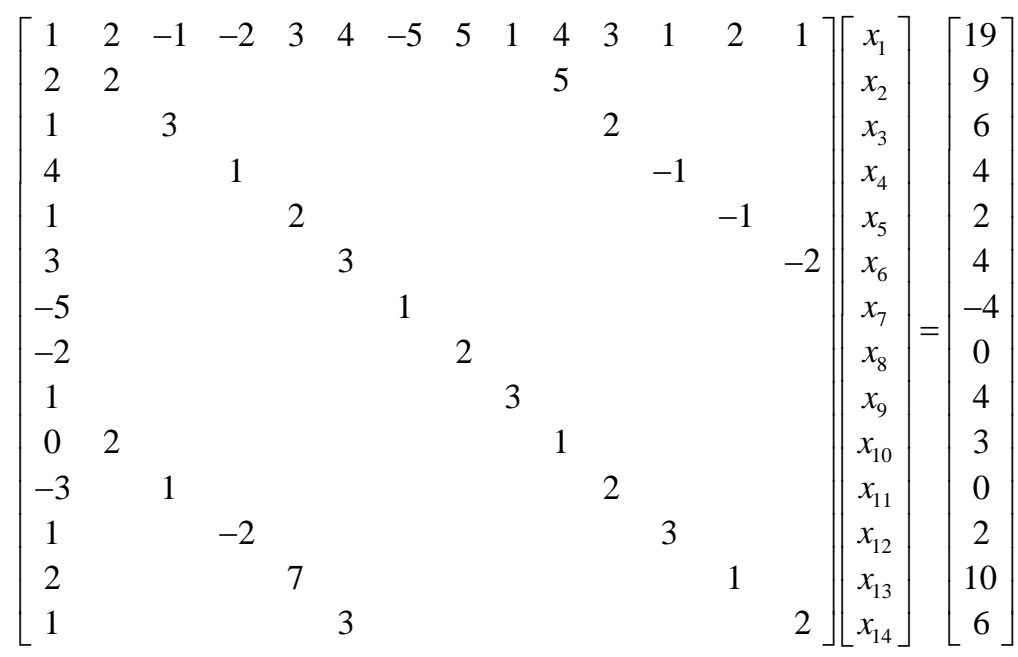


Solution: In this example, we have $n=14$ and $k=8$.

Applying the $k$-DBTLSys algorithm gives:

- $\boldsymbol{e}=\left[\begin{array}{llllllllllllll}19 / 6 & -8 & 2 & 1 / 3 & 9 & 6 & 1 & 2 & 3 & 1 & 2 & 3 & 1 & 2\end{array}\right]$ (step 1).

- $\boldsymbol{q}^{t}=\left[\begin{array}{lllllllllllll}-6 & -5 / 2 & -4 / 3 & -11 & 5 / 2 & -5 & 5 & 1 & 4 & 3 & 1 & 2 & 1\end{array}\right]$ (step 2).

- $\boldsymbol{p}=\left[\begin{array}{lllllllllllll}-1 / 4 & 2 & 13 & 1 / 3 & 2 / 3 & -5 & -1 & 1 / 3 & 0 & -3 / 2 & 1 / 3 & 2 & 1 / 2\end{array}\right]^{t} \quad$ (step 3).

- $\boldsymbol{w}=\left[\begin{array}{llllllllllllll}1 & 3 / 4 & 3 & 14 & 4 / 3 & 5 / 3 & -4 & 0 & 4 / 3 & 3 & 0 & 2 / 3 & 10 & 3\end{array}\right]^{t}$ (step 4).

- The solution is $\quad \boldsymbol{x}=\left[\begin{array}{llllllllllllll}1 & 1 & 1 & 1 & 1 & 1 & 1 & 1 & 1 & 1 & 1 & 1 & 1 & 1\end{array}\right]^{t}$ (step 5).

\section{References}

[1] El-Mikkawy, M. (2003) A Note on a Three-Term Recurrencefor a Tridiagonal Matrix. Applied Mathematics and Computation, 139, 503-511. http://dx.doi.org/10.1016/S0096-3003(02)00212-6

[2] El-Mikkawy, M. (2004) On the Inverse of a General Tridiagonal Matrix. Applied Mathematics and Computation, 150, 669-679. http://dx.doi.org/10.1016/S0096-3003(03)00298-4

[3] El-Mikkawy, M. and Karawia, A. (2006) General Tridiagonal Matrices. Applied Mathematics Letters, 19, $712-720$. http://dx.doi.org/10.1016/j.aml.2005.11.012

[4] El-Mikkawy, M. and Rahmo, E. (2008) A New Recursive Algorithm for Inverting Tridiagonal and Anti-Tridiagonal Matrices. Applied Mathematics and Computation, 204, 368-372. http://dx.doi.org/10.1016/j.amc.2008.06.053

[5] El-Mikkawy, M. and Sogabe, T. (2010) A New Family of k-Fibonacci Numbers. Applied Mathematics and Computation, 215, 4456-4461. http://dx.doi.org/10.1016/j.amc.2009.12.069

[6] Jia, J., Sogabe, T. and El-Mikkawy, M. (2013) Inversion of $k$-Tridiagonal Matrices with Toeplitz Structure. Computers \& Mathematics with Applications, 65, 116-125. http://dx.doi.org/10.1016/j.camwa.2012.11.001

[7] El-Mikkawy, M. and Atlan, F. (2014) A Novel Algorithm for inverting a General k-Tridiagonal Matrix. Applied Mathematics Letters, 32, 41-47. http://dx.doi.org/10.1016/j.aml.2014.02.015

[8] El-Mikkawy, M. (2012) A Generalized Symbolic Thomas Algorithm. Applied Mathematics, 3, 342-345. http://dx.doi.org/10.4236/am.2012.34052

[9] El-Mikkawy, M. (2004) A Fast Algorithm for Evaluating nth Order Tri-Diagonal Determinants. Journal of Computational and Applied Mathematics, 166, 581-584. http://dx.doi.org/10.1016/j.cam.2003.08.044

[10] El-Mikkawy, M. (2005) A New Computational Algorithm for Solving Periodic Tri-Diagonal Linear Systems. Applied Mathematics and Computation, 161, 691-696. http://dx.doi.org/10.1016/j.amc.2003.12.114

[11] El-Mikkawy, M., El-Shehawy, M. and Shehab, N. (2015) Solving Doubly Bordered Tridiagonal Linear Systems via Partition. Applied Mathematics, 6, 967-978. http://dx.doi.org/10.4236/am.2015.66089 